\title{
Disminución de enfermedades infecciosas intestinales relacionada al acceso a servicios de agua y desagüe en el Perú, 2002-2009
} Reduction of infectious intestinal diseases related to access to water and sewer services in Peru, 2002-2009

\author{
Fernando Arenas-Significación ${ }^{1,2,3}$, Carlos Gonzales-Medina ${ }^{1,2}$ \\ Estudiante de Medicina Humana, Facultad de Medicina de San Fernando. Universidad Nacional Mayor de San Marcos (UNMSM), Lima-Perú. \\ ${ }^{2}$ Asociación para el Desarrollo de la Investigación en Ciencias de la Salud (ADIECS), Lima-Perú. \\ ${ }^{3}$ Miembro de la Comisión Permanente de Investigación de la Facultad Medicina Humana, UNMSM, Lima-Perú.
}

\begin{abstract}
Resumen
Introducción: En el Perú, las enfermedades infecciosas intestinales ocupan el tercer lugar en causas de morbilidad registradas en consulta externa. Por otro lado, el acceso a agua y desagüe existió en $74,7 \%$ y $62,8 \%$, respectivamente. Objetivos: Determinar la correlación del acceso de agua potable y desagüe con las consultas por enfermedades infecciosas intestinales. Diseño: Estudio ecológico de series de tiempo. Institución: Facultad de Medicina de San Fernando, Universidad Nacional Mayor de San Marcos, Lima, Perú. Material: Registros del Ministerio de Salud y del Instituto Nacional de Estadística e Informática, Lima, Perú. Métodos: A partir de los registros del Ministerio de Salud (MINSA) de las causas de morbilidad en consulta externa y datos del Instituto Nacional de Estadística e Informática (INEI), se realizó un estudio ecológico de serie de tiempo, del periodo 2002 al 2009. Se analizó los resultados con el coeficiente rho de Spearman. Principales medidas de resultados: Coeficientes de correlación. Resultados: Se obtuvo un rho $=-0,810$ y $p=0,015$ para la relación entre acceso a agua potable y enfermedades infecciosas intestinales. Se obtuvo un rho $=-0,714$ y $p=$ 0,047 para la relación entre el acceso a desagüe y enfermedades infecciosas intestinales. Interpretación: Se encontró correlación entre el porcentaje de acceso de agua potable y desagüe y el porcentaje de consultas externas por enfermedades infecciosas intestinales, en el periodo del 2002 al 2009, en el Perú.
\end{abstract}

Palabras clave: Abastecimiento de agua, alcantarillado, diarrea.

Abstract

Background: In Peru, intestinal infections rank third as cause of morbidity registered in outpatient settings. On the other hand access to water and sewage was respectively $74.7 \%$ and $62.8 \%$. Objectives: To determine correlation of drinking water and sewage access and consultations for intestinal infectious diseases. Design: Ecological study of time series. Setting: Faculty of Medicine San Fernando, Universidad Nacional Mayor de San Marcos, Lima, Peru. Material: Records from the Ministry of Health and Statistics and data from the Statistics and Informatics National Institute, Lima, Peru. Methods: A time series study was conducted based on records of the Ministry of Health on causes of morbidity in outpatients and data from the Statistics and Informatics National Institute records during the period 2002-2009. Results were analyzed using Spearman's rho coefficient. Main outcome measures: Correlation coefficient. Results: An rho $=-0.810, p=0.015$ was obtained for access to drinking water and infectious intestinal diseases relation, and an $r=-0.714 p=0.047$ was obtained for access to sewage and infectious intestinal diseases relationship. Interpretation: It was found correlation between percentage of access to drinking water and drainage and percentage of outpatient visits for infectious intestinal diseases in the period 2002-2009 in Peru.

Key words: Water supply, sewage, diarrhea.

An Fac med. 2011;72(4):245-8

\section{INTRODUCCIÓN}

A nivel mundial, las enfermedades infecciosas son una causa importante de morbimortalidad. Dentro de este grupo, las enfermedades infecciosas intestinales representan el grupo con la más alta incidencia. Según las estimaciones para el año 2004 de la Organización Mundial de la Salud (OMS), se informó a nivel mundial un total de 4620419 episodios nuevos de enfermedades diarreicas, y en América se encontró un total de 539223 de episodios nuevos ${ }^{(1)}$. Se ha estimado la incidencia de enfermedades diarreicas en los Estados Unidos de América en 0,65 episodios por persona por año ${ }^{(2)}$.

Las enfermedades infecciosas intestinales (EII) en el Perú constituyen un problema de salud pública, ocupando el 2009 el tercer lugar en las causas de morbilidad específica registradas en consulta externa del MINSA ${ }^{(3)}$.
Según la OMS, en el 2008, el 13\% de la población mundial no tenía acceso a fuentes de agua potable. Asimismo, $17 \%$ de la población no contaba con medios de disposición de excretas. La proporción de personas con acceso a agua potable a nivel mundial aumentó de $77 \%$ a $87 \%$ en el periodo 1990 a 2008. Por otro lado, durante el periodo 1990 a 2006 cerca de 1000 millones de personas obtuvieron facilidades para la disposición de excretas ${ }^{(4)}$. 
El promedio de acceso a servicio de agua potable y saneamiento en América, en el año 2008, fue $96 \%$ y $87 \%$, respectivamente ${ }^{(5)}$. En Perú, en 2009, el porcentaje de hogares con acceso a agua potable y desagüe por red pública fue $74,7 \%$ y $62,8 \%$, respectivamente ${ }^{(6)}$.

El mejoramiento en el acceso y la calidad de los servicios de agua y desagüe han mostrado disminuir el riesgo de enfermedades diarreicas ${ }^{(7)}$. En una revisión sistemática se halló que las intervenciones dirigidas a la suplementación de agua y mejoramiento de la disposición de excretas disminuyeron $25 \%$ y $32 \%$ el riesgo, respectivamente ${ }^{(7)}$.

El objetivo del presente estudio fue determinar la correlación de la proporción de hogares con acceso a servicios de agua potable y desagüe por red pública con el porcentaje de consultas externas, registradas por el MINSA, por enfermedades infecciosas intestinales en el periodo de 2002 a 2009, en el Perú.

\section{MÉTODOS}

Se realizó un estudio ecológico de series de tiempo, considerando el periodo de 2002 a 2009.

Se efectuó un análisis secundario de datos publicados por el INEI y el MINSA.

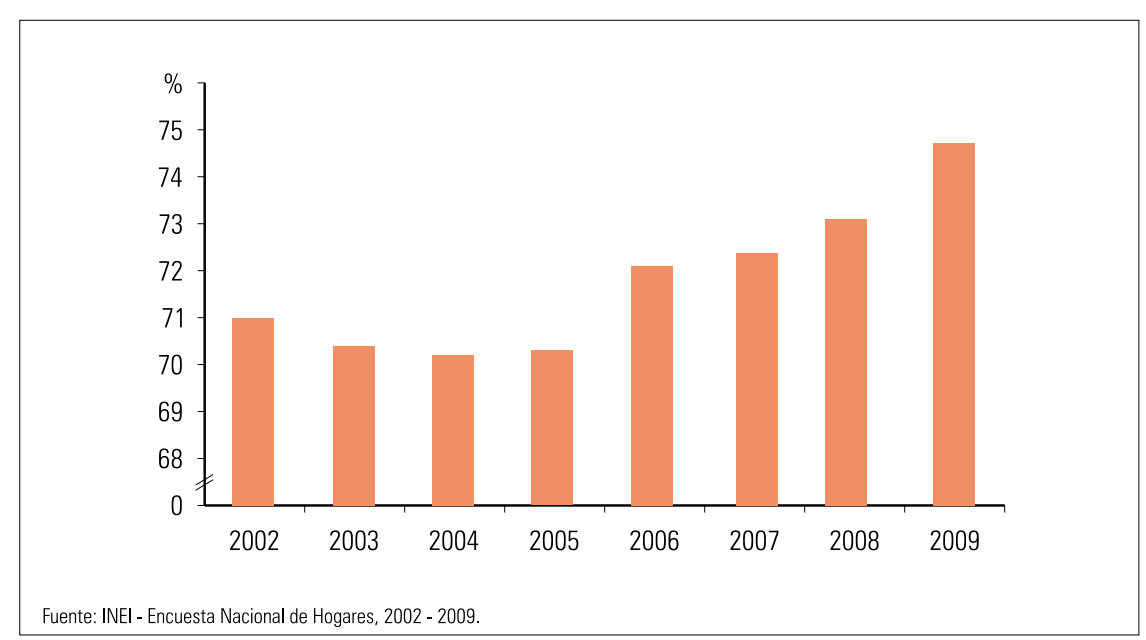

Figura 1. Porcentaje de hogares con acceso a agua potable en el Perú, $2002-2009$.

Los datos de las variables acceso a agua potable y desagüe fueron obtenidos de los registros publicados en la Encuesta Nacional de Hogares 2002. 2009, disponibles en la página oficial del INEI, en la sección de indicadores sociales de Perú en cifras ${ }^{(6)}$.

Se obtuvo los datos de la variable consultas por EII de los registros publicados de la Oficina General de Estadística e Informática del MINSA disponibles en su página oficial, en la sección de indicadores por departamentos del área de estadística ${ }^{(3)}$.

Las variables acceso a agua potable y desagüe fueron evaluadas mediante los porcentajes de hogares con acceso a agua potable y servicio de desagüe por red pública, respectivamente. En el caso de acceso a agua potable, se consideró los hogares con red pública dentro de la vivienda, red pública fuera de la vivienda pero dentro del edificio, y pilón de uso público. Se obtuvo los porcentajes desde el año 2002 al 2009.

En la variable enfermedad infecciosa intestinal se consideró a los pacientes con la codificación A00 a A09, según la clasificación internacional de enfermedades (CIE) ${ }^{(8)}$. En este grupo se incluyó cólera (A00), fiebre tifoidea y paratifoidea (A01), otras infecciones por Salmonella (A02), shigellosis (A03), otras infecciones intestinales bacterianas (A04), otras intoxicaciones alimentarias bacterianas no clasificadas en otros

.$$
\text { ent }
$$
en los siguientes años $2005(70,3 \%)$, $2006(72,1 \%), 2007(72,4 \%)$ y 2008 (73,1\%) (figura 1).

En este mismo periodo se registró un variación del porcentaje de hogares con acceso a desagüe por red pública de $53,3 \%(2002)$ a $62,8 \%$ (2009). Hubo una disminución en el año 2003 $(52,5 \%)$ y se presentó un incremento gradual en los siguientes años 2004 (54,2\%), 2005 (55,5\%), $2006(57,1 \%)$, $2007(58,8 \%)$ y $2008(60,9 \%)$ (figura 2).

Según el registro del MINSA, entre 2002 y 2009 se observó una disminución 


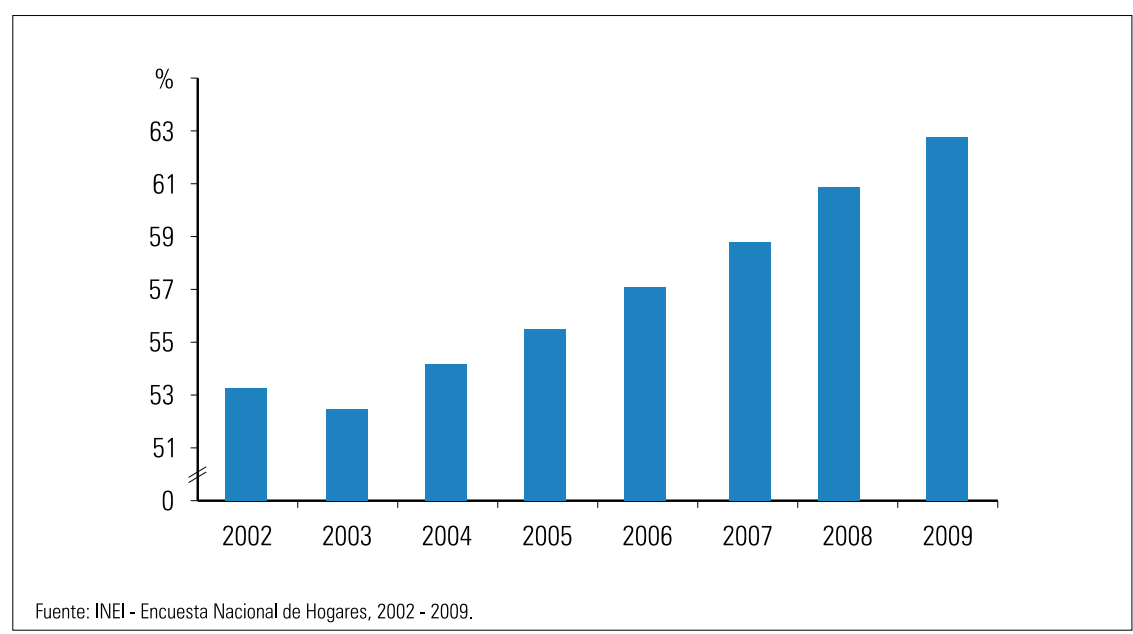

Figura 2. Porcentaje de hogares con acceso a servicio de desagüe por red pública en el Perú, $2002-2009$.

en el porcentaje de consultas externas por EII registradas, de 6,3\% (2002) a $5,2 \%$ (2009). Existió un incremento en el año 2003 (6,9\%), disminuyó el 2004 $(6,6 \%)$ y alcanzó su punto más alto el 2005 (7,0\%). Finalmente, se halló disminución gradual en los siguientes años $2006(6,8 \%), 2007(6,0 \%)$ y 2008 $(5,5 \%)$ (figura 3$)$.

Se estableció una correlación inversa estadísticamente significativa entre el porcentaje de hogares con acceso a servicio de agua potable y el porcentaje de consultas por EII. Por lo tanto, a mayor porcentaje de hogares con acceso a agua potable menor porcentaje de consultas por EII (rho $=-0,810 ; p=$ $0,015)$ (figura 4).

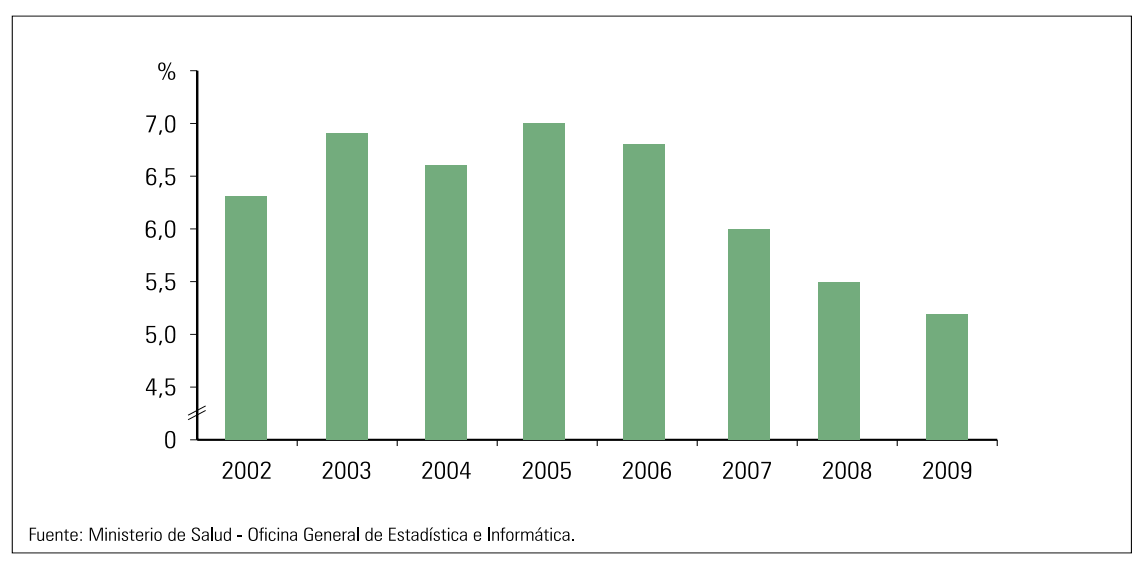

Figura 3. Porcentaje de consultas de externas por enfermedades infecciosa intestinales en el Perú, $2002-2009$.
En un estudio ecológico peruano se demostró la influencia del acceso a servicios de agua y desagüe en las atenciones por EII. Se concluyó que estas variables explican el $40,8 \%$ y $25,1 \%$ de las consultas en la población total y en los menores de 5 años ${ }^{(9)}$.

Se ha encontrado que la suplementación de agua potable disminuye las consultas por de EII y que existen otros factores que modifican el riesgo de infecciones intestinales. Dentro de estos factores se encuentran el sexo, la estación, el ingreso familiar, la fuente de agua y el uso de cloro ${ }^{(10)}$.

Cairncross y col. ${ }^{(11)}$ mostraron, mediante una revisión sistemática, que la implementación de medidas para la prevención de EII -como el lavado de manos, la mejora en la calidad del servicio de agua y desagüe- disminuyen el riesgo en $48 \%, 17 \%$ y $36 \%$, respectivamente. Existe evidencia de la relación del consumo de agua no potable e infecciones intestinales. Sin embargo, los estudios tienen metodologías diferentes y no hacen posible su agregación para la evaluación del riesgo del consumo con la datos disponibles ${ }^{(12)}$.

Las EII son prevenibles y por ello las medidas dirigidas a evitar su propagación deben ser priorizadas, pues representan un costo importante para el sistema de salud. Aunque no se ha estimado los costos de tratamiento en el Perú, existen datos publicados de los Estados Unidos. Se estimó que entre 2001 y 2005 se tuvo un total de 15420 hospitalizaciones ${ }^{(13)}$ y que el costo de servicios de salud fue 1,4 trillones de dólares ${ }^{(14)}$.

Debido a que estos resultados se han basado solo en datos del MINSA, sin tomar en cuenta las consultas en otros servicios de salud, es posible que haya una subestimación de las consultas por EII. Se debe tener precaución al interpretar los resultados de este tipo de estudios, pues se puede incurrir en la falacia ecológica al intentar generalizar los resultados del nivel poblacional al nivel individual. También, se debe tener en cuenta que no se ha estudiado 


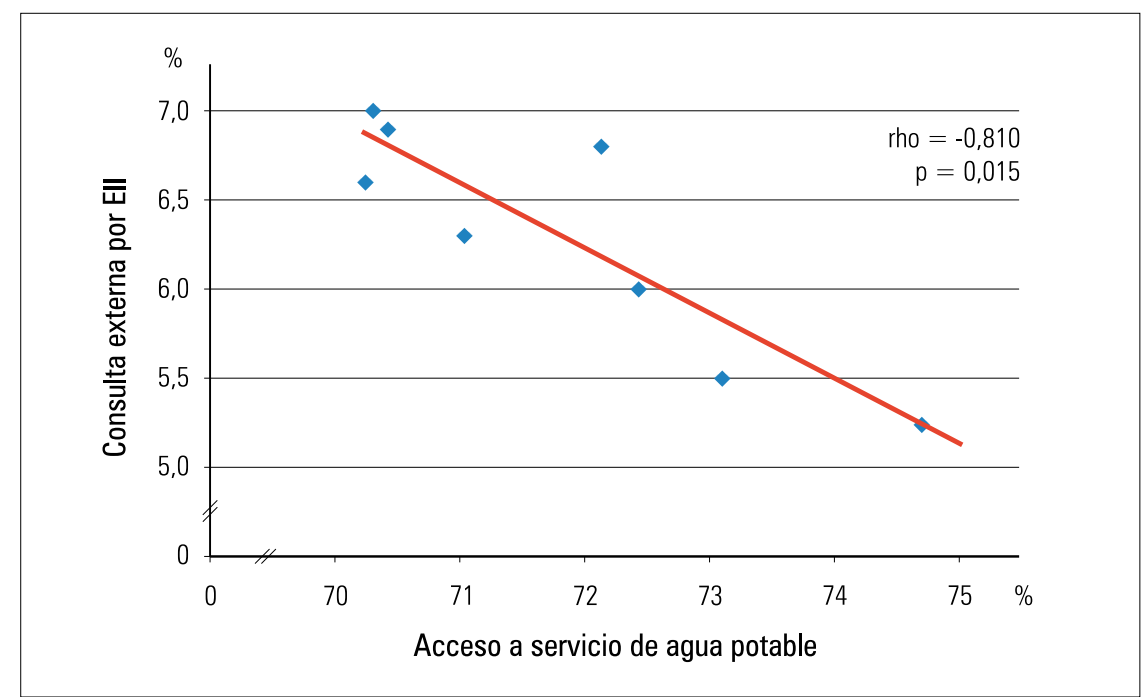

Figura 4. Correlación entre el porcentaje de hogares con acceso a servicio de agua potable y el porcentaje de consultas externas por enfermedades infecciosas intestinales.

otros factores que podrían modificar la propagación de la enfermedad ${ }^{(15)}$.

Además, se ha utilizado el porcentaje de consultas por EII como indicador en lugar de la tasa de EII, pues no se tuvo al alcance este último. Asimismo, se ha trabajado con los porcentajes de acceso en lugar de cifras absolutas por las diferencias del tamaño de la población en cada año. A pesar de ello, los estudios ecológicos constituyen una herramienta útil y rápida para orientar las estrategias sanitarias que deben ser aplicadas ${ }^{(16)}$.
Se concluye que existe correlación inversa entre el porcentaje de acceso a agua y desagüe y las consultas por EII en el periodo del 2002 al 2009, lo cual constituye un factor importante a considerar en las futuras estrategias preventivas de estas enfermedades.

\section{REFERENCIAS BIBLIOGRÁFICAS}

1. World Health Organization. Global Health Observatory Data Repository [página web en Internet]. Geneva: WHO [citado el 29 de junio de 2011]. Disponible en: http://apps.who.int/ghodata/.

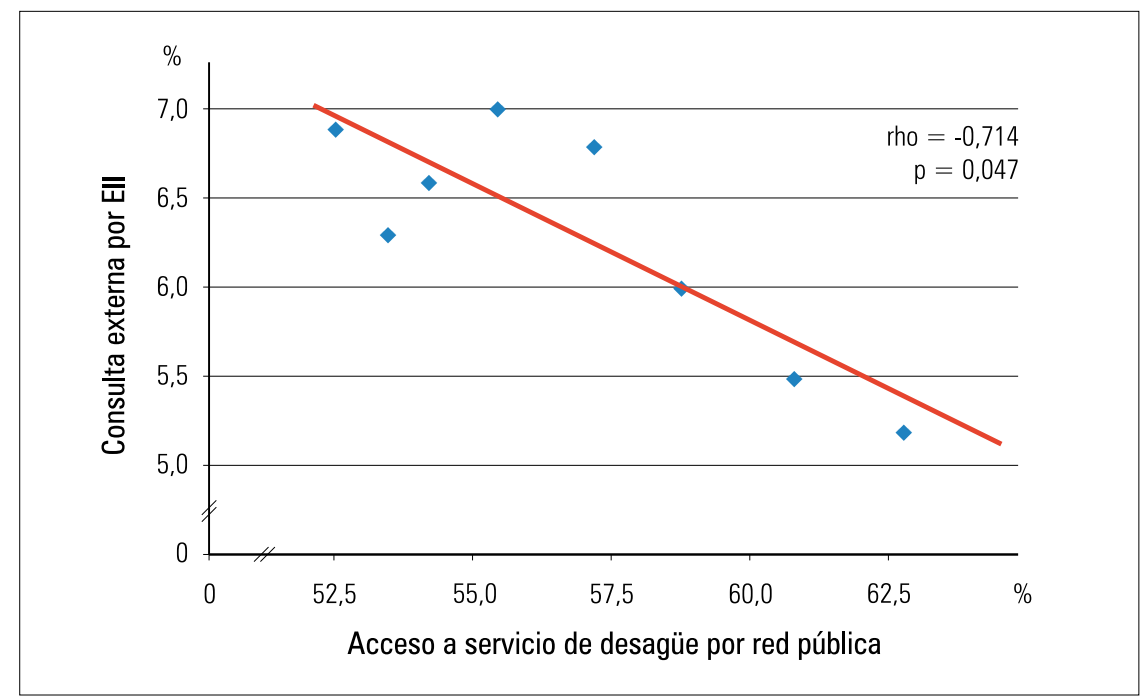

Figura 5. Correlación entre el porcentaje de hogares con acceso a servicio de desagüe por red pública y el porcentaje de consultas externas por enfermedades infecciosas intestinales.

2. Roy SL, Scallan E, Beach MJ. The rate of acute gastrointestinal illness in developed countries. J Water Health. 2006;4(Suppl 2):31-69.

3. Ministerio de Salud del Perú. Principales causas de morbilidad: consulta externa [página web en Internet]. Perú: MINSA [citado el 29 de junio de 2011]. Disponible en: http://www.minsa.gob.pe/ portada/estadistica.asp.

4. World Health Organization. Water and sanitation [página web en Internet]. Geneva: WHO [citado el 29 de junio de 2011]. Disponible en: http://www. who.int/gho/mdg/environmental_sustainability/en/ index.html.

5. World Health Organization. Health-related Millennium Development Goals. En: World health statistics 2010. Geneva: WHO; 2010.

6. Instituto Nacional de Estadistica e Informática. Perú en cifras: hogar [página web en Internet]. Perú: INEI [citado el 29 de junio de 2011]. Disponible en: http:// www.inei.gob.pe/.

7. Fewtrell L, Kaufmann RB, D Kay, Enanoria W, HaIler L, Colford JM. Water, sanitation, and hygiene interventions to reduce diarrhoea in less developed countries: a systematic review and meta-analysis. Lancet Infect Dis. 2005;5:42-52.

8. World Health Organization. Certain infectious and parasitic diseases. En: International Statistical Classification of Diseases and Related Health Problems. 7th revision. Geneva: WHO; 2007.

9. Ramos W, Valdez W, Miranda J, Tovar JC. Influencia del acceso a servicios de agua y desagüe sobre las atenciones por enfermedad diarreica aguda en establecimientos del Ministerio de Salud. Estudio ecológico: Perú, enero a diciembre de 2007. Rev Peru Epidemiol. 2010;14(1):1-7

10. Teschke K, Bellack N, Shen H, Atwater J, Chu R, Koehoorn $\mathrm{M}$, et al. Water and sewage systems, socio-demographics, and duration of residence associated with endemic intestinal infectious diseases: A cohort study. BMC Public Health. 2010;10:767.

11. Cairncross S, Hunt C, Boisson S, Bostoen K, Curtis $\checkmark$, Fung I, et al. Water, sanitation and hygiene for the prevention of diarrhea. Internat $\mathrm{J}$ Epidemiol. 2010;39:i193-i205.

12. Mann A, Tam C, Higgins CD, Rodrigues LC. The association between drinking water turbidity and gastrointestinal illness: a systematic review. BMC Public Health. 2007;7:256.

13. Buzby J, Roberts T. The economics of enteric infections: human foodborne disease costs. Gastroenterol. 2009;136:1851-62.

14. Roberts T. WTP estimates of the societal cost of US food-borne illness. Am J Agric Econ. 2007;5:1183-8.

15. Diez Roux A. La necesidad de un enfoque multinivel en epidemiología. Región y Sociedad. 2008;2(2):77-91.

16. Borja-Aburto VH. Estudios ecológicos. Salud Pública de México. 2000;42(6):533-8.

Artículo recibido el 29 de junio de 2011 y aceptado para publicación el 16 de agosto de 2011.

\section{Correspondencia:}

Arenas-Significación Fernando

Teléfonos: 4988003 / 994929088

Correos: fer_06141@hotmail.com,

fer_medicine@yahoo.es. 